\section{EFEITOS AGUDOS NO DESEMPENHO DO SALTO VERTICAL APÓS O AGACHAMENTO COM BANDA ELÁSTICA DE JOELHO}

\author{
ACUTE EFFECTS ON PERFORMANCE OF THE VERTICAL JUMP AFTER BACK SQUAT WITH \\ KNEE WRAPS \\ EFECTOS AGUDOS EN EL DESEMPEÑO DEL SALTO VERTICAL DESPUÉS DE SENTADILLA CON \\ BANDA ELÁSTICA DE RODILLA
}

Willy Andrade Gomes'

(Educador Físico)

Josinaldo Jarbas da Silva'

(Educador Físico)

Enrico Gori Soares ${ }^{1}$

(Educador Físico)

Erica Paes Serpa

(Educadora Física)

Daniel Alves Corrêa'

(Educador Físico)

Guanis de Barros Vilela Junior' (Educador Físico)

Charles Ricardo Lopes ${ }^{1,2}$

(Educador Físico)

Paulo Henrique Marchetti ${ }^{1,3}$

(Educador Físico)

1. Universidade Metodista de Piracicaba, Faculdade de Ciências da Saúde, Grupo de Pesquisa em Performance Humana, Piracicaba, SP, Brasil.

2. Faculdade Adventista de Hortolândia, Hortolândia, SP, Brasil. 3. Universidade de São Paulo, SP, Brasil, Faculdade de Medicina, Hospital das Clinicas, Instituto de Ortopedia e Traumatologia, São Paulo, SP, Brasil.

\section{Correspondência:}

Universidade Metodista de

Piracicaba, Faculdade de Ciências da Saúde, Programa de Pós-Graduação em Ciências do Movimento Humano, Grupo de Pesquisa em Performance Humana.

Rodovia do Açúcar Km 156, Bloco 7 , Sala 39, Taquaral. 13423-070, Piracicaba, SP, Brasil.

dr.pmarchetti@gmail.com

\section{RESUMO}

Introdução: A banda elástica pode afetar o desempenho durante exercícios de alta intensidade, reduzindo a ativação muscular, o que pode influenciar o efeito de potencialização pós-ativação, que depende de alta intensidade para o exercício subsequente que visa potência. Objetivo: Verificar os efeitos agudos no desempenho do salto com contramovimento (SCM) após o agachamento em alta intensidade com e sem o uso da banda elástica de joelhos em sujeitos treinados em força. Métodos: Participaram do estudo 14 homens (idade: $24 \pm 4$ anos, estatura: $176 \pm 6 \mathrm{~cm}$, massa corporal: $81 \pm 11$ kg, 1RM: $107 \pm 30 \mathrm{kgf}$ ), treinados em força (>3 anos). Foram realizados três SCM antes e após três meio-agachamentos a $90 \%$ de 1RM nas condições com e sem a banda elástica de joelhos. Durante o SCM foram avaliados ativação muscular (IEMG) do vasto lateral (VL), glúteo máximo (GM), tempo de salto (TS) e impulso (IMP) por meio da força vertical de reação do solo (FRSv). Resultados: Os resultados mostraram que para o TS foi verificada diferença significante entre as condições pós-meio-agachamento (com e sem banda) $(P=0,044, \mathrm{TE}=1,02)$, sendo que os maiores valores foram observados para a condição sem banda. Para a IEMG de VL foi verificada diferença significante entre as condições pré e pós o meio-agachamento (com banda) $(P=0,029, T E=1,68$ ), sendo que os maiores valores foram observados para a IEMG com banda. Para a IEMG de GM e para o IMP não foram verificadas diferenças significantes entre as condições. Conclusão: Após a utilização da banda elástica de joelhos durante o exercício agachamento, ocorreu uma redução na participação do vasto lateral durante o salto com contramovimento, afetando o tempo de contato, mas não a produção de impulso, e consequente desempenho.

Palavras-chave: força muscular, desempenho atlético, treinamento de resistência.

\section{ABSTRACT}

Introduction: Knee wraps can affect the muscular performance during high-intensity exercises by reducing muscle activation, which may influence the effect of post-activation potentiation, which depends on high intensity for the following power exercise. Objective: To investigate the acute effects on the performance with counter-movement jump (CMJ) after high-intensity squat with and without the use of knee wraps in resistance trained subjects. Methods: The study included 14 men (aged $24 \pm 4$ years, height: $176 \pm 6 \mathrm{~cm}$, weight: $81 \pm 11 \mathrm{~kg}, 1 \mathrm{RM}: 107 \pm 30 \mathrm{kgf}$ ) resistance trained ( $>3$ years). Three SCM were performed before and after three back squats at $90 \%$ of 1 RM with and without the knee wraps. During the CMJ muscle activation (IEMG) of the vastus lateralis (VL) and gluteus maximus (GM), jump time (JT), and impulse (IMP) through the force of vertical ground reaction (FRSV) were evaluated. Results: The results show statistically significant differences for JT between post-back-squat conditions (with and without wrap) ( $P=0.044, T E=1.02)$, whereas the highest values were observed without knee wrap. For IEMG of VL significant difference between pre and post-conditions after back squat (with knee wrap) $(P=0.029, T E=1.68)$ was observed, and the highest values were observed for the IEMG with knee wrap. No significant differences between conditions were observed for IEMG of GM and IMP. Conclusion: After using the knee wraps during the squat exercise, there was a reduction in the involvement of the vastus lateralis during the jump with counter-movement, affecting the contact time, but not the production of impulse and consequent performance.

\section{Keywords: muscle strength, athletic performance, resistance training.}

\section{RESUMEN}

Introducción: La banda elástica en la rodilla puede afectar al rendimiento durante ejercicios de alta intensidad, reduciendo la activación muscular, lo que pueden influir en el efecto de la potenciación post-activación, que depende de alta intensidad para el próximo ejercicio que tiene como objetivo la potencia. Objetivo: Investigar los efectos agudos en el rendimiento en salto con contramovimiento (SCM) después de sentadilla de alta intensidad con y sin el uso de bandas elásticas en las rodillas en sujetos entrenados en fuerza. Métodos: El estudio incluyó a 
14 hombres (edad $24 \pm 4$ años, altura: $176 \pm 6$ cm., masa corporal: $81 \pm 11$ Kg., 1RM: $107 \pm 30$ kgf), entrenados en fuerza (> 3 años). Tres SMC fueron realizados antes y después de las tres medias sentadillas al $90 \%$ de 1RM en las condiciones con y sin las bandas elásticas en las rodillas. Durante el SMC se evaluó la activación muscular (IEMG) del vasto lateral (VL), glúteo mayor (GM), el tiempo de salto (TS) y el impulso (IMP) a través de la fuerza de reacción vertical del suelo (FRSV). Resultados: Los resultados mostraron que para el TST hubo diferencia significativa entre las condiciones post-media sentadilla (con y sin banda) $(P=0,044, T E=1,02)$, mientras que se observaron los valores más altos para la condición sin la banda. Para IEMG de VL hubo diferencias estadísticamente significativas entre las condiciones pre y post la media sentadilla (con banda) $(P=0,029, T E=1,68)$, y se observaron valores más altos para IEMG con la banda. Para IEMG de GM y el IMP se encontraron diferencias significativas entre las condiciones. Conclusión: Después de usar la banda elástica de rodillas durante el ejercicio de sentadilla, hubo una reducción en la participación del vasto lateral durante el salto con contramovimiento, afectando el tiempo de contacto, pero no la producción de impulsos, y el consiguiente rendimiento.

Palabras clave: fuerza muscular, rendimiento atlético, entrenamiento de fuerza.

\section{INTRODUÇÃO}

A banda elástica de joelhos é um equipamento comumente utilizado para aumentar o desempenho no exercício agachamento de diversas modalidades esportivas que exijam força e potência. Em teoria, a melhora do desempenho produzido pela banda elástica se baseia no efeito mecânico dos seus componentes elásticos, os quais aumentam a capacidade de carregamento, chamado de "carry-over", facilitando o levantamento de cargas na fase concêntrica do movimento'. Gomes et al. ${ }^{2}$ compararam o efeito de diferentes modelos de rigidez de banda elástica (Soft e Hard) de joelhos durante o agachamento isométrico máximo e observaram aumentos nos picos de força de 21 e 22\% respectivamente. Harman e Frykman³ verificaram um aumento de $25 \%$ em sujeitos treinados durante o agachamento isométrico com banda elástica.

Quanto ao efeito da banda elástica de joelhos no desempenho do exercício agachamento, o estudo de Gomes et al. ${ }^{4}$ verificou alterações no padrão de recrutamento muscular de vasto lateral e glúteo máximo durante o agachamento dinâmico com banda elástica de joelhos, em diferentes intensidades (60 e 90\% de 1RM), sendo observada redução da ativação muscular do vasto lateral a 90\% de 1RM. Em estudo realizado por Lake et al. ${ }^{5}$ foram verificadas alterações no padrão de movimento do exercício agachamento com o uso da banda elástica de joelhos. Os resultados mostraram uma redução de 39\% do deslocamento horizontal da barra na fase descendente, além de uma redução do tempo de duração da fase descendente absoluta e um aumento da potência pico quando os sujeitos utilizaram a banda elástica. Concluindo que as propriedades mecânicas da banda alteraram a técnica e possivelmente os músculos alvo do exercício. Por outro lado, o exercício agachamento, sem a banda elástica, parece afetar positivamente diferentes movimentos e exercícios, quando associado com a correta sobrecarga. Fukutani et al. ${ }^{6}$ verificaram melhora do desempenho de saltos verticais quando esses foram precedidos pela realização do exercício agachamento em diferentes intensidades (45, 60, 75 e 90\% de 1RM) após intervalo de um minuto. Essa estratégia é conhecida como potencialização pós-ativação (PPA), fenômeno caracterizado por um aumento agudo na força e potência, podendo aumentar o desempenho físico ${ }^{7,8}$. Dentro da literatura científica, algumas pesquisas vêm tentando identificar métodos que aperfeiçoem a PPA ${ }^{8-11}$, sendo que os principais mecanismos responsáveis pela PPA parecem estar associados à fosforilação da cadeia leve reguladora de miosina, aumento da afinidade do $\mathrm{Ca}^{2+}$ a troponina, aumento do recrutamento de unidades motoras de ordem mais elevada, uma possível mudança no ângulo de penação das fibras musculares ${ }^{7,12}$, além do equilíbrio entre a fadiga instaurada e a potencialização gerada pela atividade anterior ${ }^{8}$. Este equilíbrio é afetado por fatores que incluem a experiência de treinamento dos sujeitos (atletas de alto nível treinados em força ou sujeitos destreinados), duração do período de atividade pós-condicionamento, e da intensidade da sobrecarga ${ }^{8}$.

Baseado no efeito carry-over, durante o uso da banda elástica de joelhos no exercício agachamento, pode-se conjecturar que o aumento da sobrecarga levantada afetaria a atividade de força/potência posterior, e consequentemente a PPA.

Portanto, o objetivo do presente estudo foi verificar os efeitos agudos no desempenho do salto com contra-movimento (SCM) após o agachamento em alta intensidade com e sem o uso da banda elástica de joelhos em sujeitos treinados em força.

\section{MATERIAIS E MÉTODOS}

O número de sujeitos $(n=14)$ foi determinado utilizando um estudo piloto previamente realizado, com indivíduos que possuíam as mesmas características das que foram empregadas no presente estudo, baseado em significância de 5\% e um poder do teste de $80 \%{ }^{13}$. Participaram do estudo 14 indivíduos do sexo masculino (idade: $24 \pm 4$ anos, estatura:

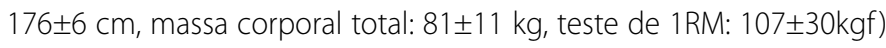
treinados em força por mais de três anos, aptos fisicamente. Todos os sujeitos eram previamente familiarizados com a banda elástica e com o exercício agachamento. Todos os sujeitos foram informados dos procedimentos experimentais, leram e assinaram o Termo de Consentimento Livre e Esclarecido aprovado pelo Comitê de Ética em Pesquisa da Universidade (protocolo no 76/12).

O estudo foi dividido em duas sessões distintas com intervalo de 48 horas entre elas. Durante a primeira sessão, foram coletados os dados antropométricos (massa, estatura), e pessoais (nível de experiência e idade). Então, os sujeitos foram submetidos a dois tipos de familiarização, sendo a primeira relacionada à técnica do meio-agachamento, e a segunda ao teste de salto com contra-movimento (SCM). A familiarização com o meio-agachamento foi realizada por meio de quatro séries de 10 repetições utilizando uma sobrecarga equivalente a $50 \%$ da massa corporal total do sujeito, nas condições com e sem a banda elástica de joelhos, de forma aleatorizada entre os sujeitos. Os sujeitos realizaram a técnica do meio-agachamento com a amplitude do movimento limitada pelo momento em que a coxa atingia a posição paralela ao solo. A distância entre os membros inferiores durante a realização do exercício agachamento foi proporcional à largura do quadril, a qual foi determinada através de avaliação visual. Após o posicionamento dos pés no solo, o local foi demarcado 
para garantir que os sujeitos mantivessem sua posição constante em todos os testes subsequentes. A segunda familiarização foi realizada a partir do teste de salto vertical máximo (SCM) realizado três vezes, e sem o uso da banda elástica. O intervalo entre as tentativas foi de três segundos. Após 10 minutos de descanso, um teste de carga máxima (1RM) foi realizado para o exercício meio-agachamento, apenas na condição sem a banda elástica de joelhos, visando determinar a intensidade da sobrecarga externa a ser utilizada no experimento (90\%1RM). A técnica para a realização do teste de 1RM se baseou nas orientações da "American Society of Exercise Physiologists"14.

\section{Protocolo experimental}

Durante a segunda sessão foi realizado um breve aquecimento utilizando o meio-agachamento, e em seguida três saltos máximos (SCM) e sequenciais, com intervalo de três segundos entre as tentativas foram realizados como condição pré-salto. Então, após três minutos, foi realizada uma série de três repetições, a 90\% de 1RM utilizando o exercício meio-agachamento em duas condições distintas: com e sem a banda elástica de joelhos. Logo após um minuto de intervalo para cada condição de meio-agachamento foram realizados mais três saltos máximos (SCM), considerada como condição pós-intervenção. O tempo de intervalo entre as condições foi de três minutos, baseado no tempo necessário para a ressíntese da creatina fosfato ${ }^{15}$. Todas as condições (meio-agachamento com e sem banda) foram aleatorizadas e os movimentos foram realizados em cadência auto-selecionada. Foi utilizada uma banda elástica de 0,02 × 0,08 × 2,00 m, composta por tecido de algodão pesado com filamentos entrelaçados de borracha elástica (Maba Murphy Confecções Ltda., Brasil). A técnica de colocação da banda elástica adotada foi em espiral, o mais apertado possível, e sendo a mesma colocada sempre pelo mesmo pesquisador, visando reduzir a variabilidade inter-avaliadores.

\section{Avaliações}

Salto com contra-movimento (SCM): A avaliação do SCM foi realizada no início e após a execução das condições de meio-agachamento (com e sem banda). Para a realização do SCM, os sujeitos foram posicionados sobre uma plataforma de força (EMG832C, EMG System Brasil, São José dos Campos, Brasil), com as mãos apoiadas sobre o quadril, para que não afetassem a propulsão dos membros inferiores. Todos os saltos foram realizados de forma máxima e sem a utilização da banda elástica de joelhos.

Eletromiografia Superficial (sEMG): Dados da atividade muscular de glúteo máximo e vasto lateral, durante os SCMs, foram adquiridos através de um eletromiógrafo integrado à plataforma de força que mensurou a força de reação do solo vertical (FRSv). Para a coleta dos dados de sEMG, foi utilizado um eletromiógrafo de quatro canais (EMG832C, EMG System Brasil, São José dos Campos, Brasil) com frequencia de amostragem de $2000 \mathrm{~Hz}$, através de um software de aquisição (DATAQ Instruments Hardware Manager, DATAQ Instruments, Inc., OH, USA). A atividade sEMG foi amplificada (amplificador bi-polar diferencial, com impedância de entrada $=2 \mathrm{M} \Omega$, e taxa de rejeição de modo comum $>100 \mathrm{~dB}(60 \mathrm{~Hz})$, ganho x100, ruído $<5 \mu \mathrm{V})$, e um conversor $\mathrm{AD}$ de 12 bits. Foram utilizados pares de eletrodos ativos de superfície com ganho de 10x, circulares, auto-adesivos, Ag/AgCl com $1 \mathrm{~cm}$ de raio, com espaçamento de $2 \mathrm{~cm}$ de centro a centro entre os eletrodos, associados à um gel condutor, sendo colocados sobre o glúteo máximo (GM) e vasto lateral (VL). Os eletrodos foram posicionados como segue: GM na porção média a 50\% entre a crista ilíaca e o trocanter maior e para o VL foi a 2/3 na linha a partir da espinha ilíaca anterior superior da porção lateral da patela ${ }^{16}$. 0 eletrodo monopolar de referência foi colocado na proeminência óssea da clavícula do lado direito dos sujeitos. Os procedimentos para a realização do CVMI se basearam nas orientações de Winter ${ }^{17}$ e foram realizados separadamente através de contrações máximas isométricas de cinco segundos (glúteo máximo: em extensão de quadril com os joelhos em semi-flexão e vasto lateral: em $90^{\circ}$ de flexão de joelhos), ambos contra uma resistência externa fixa imposta pelo avaliador.

\section{Análise dos Dados}

A força de reação do solo vertical (FRSv) advinda da plataforma de força, foi coletada durante os saltos realizados antes e após a realização do exercício agachamento (com e sem banda). Os dados foram filtrados com um filtro passa-baixa de $100 \mathrm{~Hz}$, Butterworth de quarta ordem e atraso de fase zero e normalizados pelo peso corporal total. O início e o final da fase propulsiva do salto foram definidos através dos dados da FRSv, e analisada de forma simultânea aos dados sEMG de vasto lateral e glúteo máximo. Para os dados de FRSv, foram analisados o impulso (integral força $x$ tempo) e o tempo de salto (caracterizado pelo tempo entre o início e final da fase propulsiva $)^{18}$.

Os dados sEMG foram processados através de uma rotina escrita no software Matlab (Mathworks Inc., EUA). Os dados sEMG foram definidos através da FRSv, para todas as condições realizadas. O processamento do sinal sEMG seguiu a seguinte ordem ${ }^{19}$ os sinais sEMG foram filtrados com um filtro Butterworth de $4^{\text {a }}$ ordem, passa banda entre $20-400 \mathrm{~Hz}$, e atraso de fase zero, então os dados sEMG foram processados por uma root-mean square janelada a 150 m (RMS EMG), normalizados pelo pico da CVMI e, então intergrados (IEMG). Os dados EMG foram também normalizados na base temporal, definido pelo tempo na fase propulsiva do SCM.

\section{Análise Estatística}

A normalidade e homogeneidade das variâncias foram verificadas utilizando o teste de Shapiro-Wilk e de Levene, respectivamente. Todos os dados foram reportados através da média e desvio padrão (DP) da média. ANOVA (2X2) foi utilizada com medidas repetidas, tendo como fatores a banda (com e sem o uso) e condição (pré e pós-intervenção), para comparar as diferenças nas variáveis dependentes: impulso, tempo de salto e IEMG ${ }_{\text {salto }}$ (vasto lateral e glúteo máximo). O cálculo do tamanho do efeito (TE) foi realizado através da fórmula de Cohen e os resultados se basearam nos seguintes critérios: $<0,35$ efeito trivial; 0,35-0,80 pequeno efeito; 0,80-1,50 efeito moderado; e >1,50 grande efeito, para sujeitos treinados recreacionalmente baseado em Rhea ${ }^{20}$. Significância de 5\% foi utilizada para todos os testes estatísticos, através do software SPSS versão 21.0.

\section{RESULTADOS}

Para o tempo de salto foi verificada diferença significante entre as condições pós-intervenção (com e sem banda) $(P=0,044$, TE=1,02), sendo que os maiores valores foram observados para a condição sem banda (figura 1a). Entretanto, para o impulso não foram verificadas diferenças significantes entre as condições.

Para a IEMG de vasto lateral foi verificada diferença significante entre as condições pré e pós-intervenção apenas com banda ( $P=0,029$, $\mathrm{TE}=1,68)$, sendo que os maiores valores foram observados para a condição pré-intervenção. Para a IEMG de glúteo máximo não foram verificadas diferenças significante entre as condições (figura 1b).

\section{DISCUSSÃO}

Os resultados mostraram que o uso da banda elástica de joelhos durante o meio-agachamento não afetou o desempenho do SCM para as variáveis dependentes, tempo de salto e impulso. No entanto, 


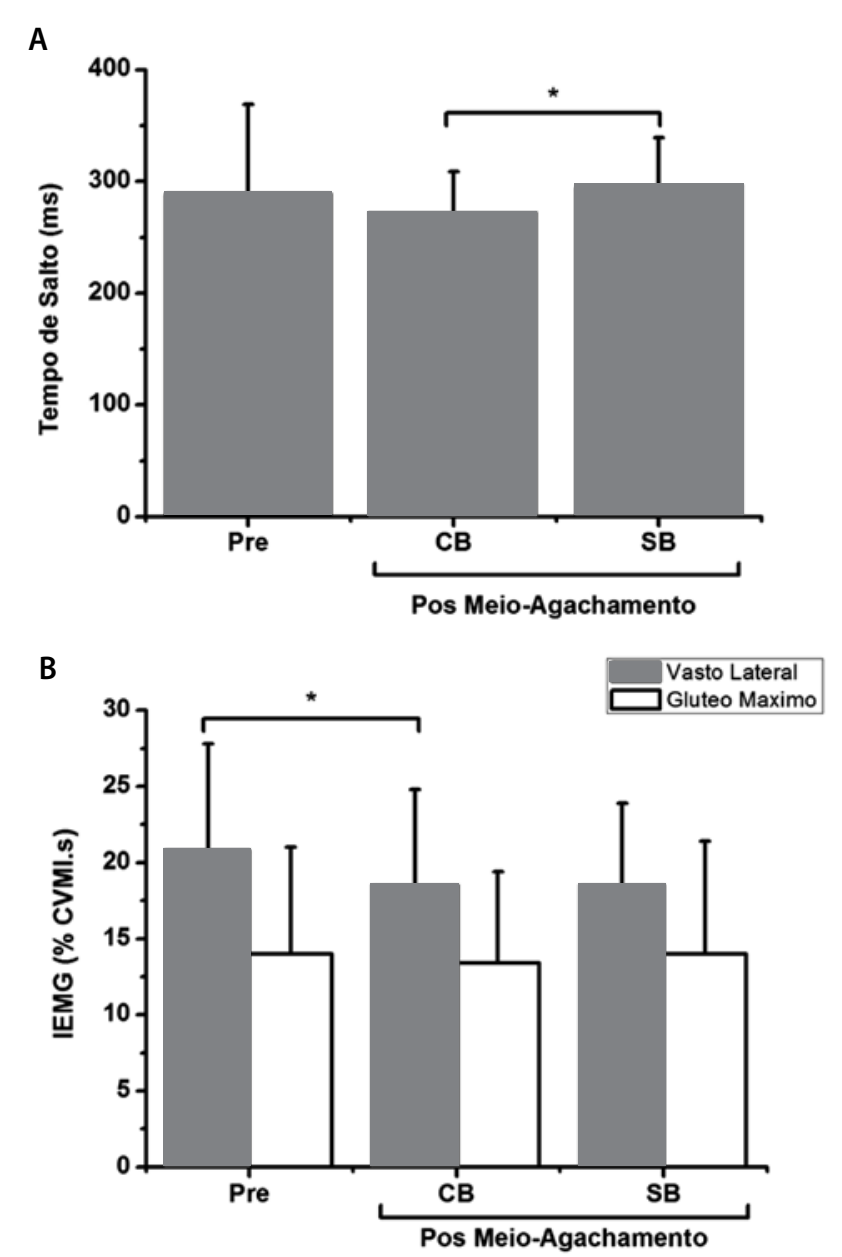

Figura 1. Média e desvio padrão (a) tempo de salto e (b) atividade muscular (IEMG) de vasto lateral e glúteo máximo, para as condições pré e pós-agachamento, com banda (CB) e sem banda (SB). ${ }^{*} \mathrm{P}<0,05$.

quando comparado o tempo de salto entre as condições (com e sem banda após o meio-agachamento à 90\% de 1RM), foi verificado um menor tempo de salto para a condição com banda. Interessantemente, este efeito ocorreu mesmo com a alteração do padrão de ativação muscular do VL, o qual apresentou redução na IEMG. No entanto, para o GM não foram observadas alterações no SCM, entre os momentos (pré e após-agachamento).

Possivelmente, com a utilização da banda elástica de joelhos durante o meio-agachamento, obteve-se o efeito carry-over, como observado em estudos prévios ${ }^{2,3,21}$ reduzindo a atividade muscular do VL, como verificado por Gomes et al. ${ }^{4}$. Entretanto, possivelmente, o efeito carry- over produzido durante o agachamento com banda elástica não afetou significantemente no impulso de salto, entre os momentos pré e pós o exercício agachamento.

Estudos prévios reportam o efeito da PPA em diferentes intervalos de recuperação entre o exercício agachamento e os saltos analisados 6,8,22-25. Tais estudos verificaram que tempos de recuperação acima de três minutos ${ }^{8,22-25}$ apresentaram aumento do desempenho de saltos, atingindo seu respectivo desempenho máximo entre quatro a nove minutos, além de poder ser afetado também pelo nível de condicionamento do sujeito $22,24,25$. Outro ponto à ser considerado é o efeito da carga, já que o presente estudo realizou três repetições a 90\% de 1RM com um intervalo de 1 minuto, em sujeitos treinados em força, o que pode ter sido insuficiente para manifestar a PPA durante o SCM.

\section{CONCLUSÃO}

Conclui-se que, após a utilização da banda elástica de joelhos durante o exercício agachamento, ocorreu uma redução na participação do vasto lateral durante o salto com contra-movimento, afetando o tempo de contato, mas não a produção de impulso, e consequente desempenho.

\section{AGRADECIMENTOS}

O presente trabalho foi realizado com apoio da Coordenação de Aperfeiçoamento de Pessoal de Nível Superior (CAPES) Brasil.

Todos os autores declararam não haver qualquer potencial conflito de interesses referente a este artigo.

\section{REFERÊNCIAS}

1. Gomes WA, Coutinho M, Marchetti PH. Revisão dos efeitos biomecânicos do uso da banda elástica no joelho durante o agachamento no levantamento básico. CPAQV. 2013;5(3):1-15.

2. Gomes WA, Serpa EP, Silva JJ, Correa DA, Silva FHDO, Neto FA, et al. Acute effects on maximal isometric force with and without knee wrap during squat exercise. Int J Sports Sci. 2014;4(2):47-9.

3. Harman E, Frykman P. The effects of knee wraps on weightlifting performance and injury. J Strength Cond Res. 1990;12:30-5.

4. Gomes WA, Brown LE, Soares EG, Silva JJ, Silva FH, Serpa ÉP, et al. Kinematic and sEMG analysis of the back squat at different intensities with and without knee wraps. J Strength Cond Res. 2015 Mar 10. [Epub ahead of print] PubMed PMID: 25763519

5. Lake JP, Carden PJ, Shorter KA. Wearing knee wraps affects mechanical output and performance characteristics of back squat exercise. J Strength Cond Res. 2012;26(10):2844-9.

6. Fukutani A, Takei S, Hirata K, Miyamoto N, Kanehisa H, Kawakami Y. Influence of the intensity of squat exercises on the subsequent jump performance. J Strength Cond Res. 2014;28(8):2236-43.

7. Ide BN, Lopes CR, Sarraipa MF. Fisiologia do treinamento esportivo: força, potência, velocidade, resistência, periodização e habilidades psicológicas. São Paulo: Phorte; 2010.

8. Wilson JM, Duncan NM, Marin PJ, Brown LE, Loenneke JP, Wilson SM, et al. Meta-analysis of postactivation potentiation and power: effects of conditioning activity, volume, gender, rest periods, and training status. J Strength Cond Res. 2013;27(3):854-9. d

9. Brandenburg JP. The acute effects of prior dynamic resistance exercise using different loads on subsequent upper-body explosive performance in resistance-trained men. J Strength Cond Res. 2005;19(2):427-32.

10. Chiu LZ, Fry AC, Weiss LW, Schilling BK, Brown LE, Smith SL. Postactivation potentiation response in athletic and recreationally trained individuals. J Strength Cond Res. 2003;17(4):671-7.

11. Farup J, Sørensen H. Postactivation potentiation: upper body force development changes after maximal force intervention. J Strength Cond Res. 2010;24(7):1874-9.

12. Tillin NA, Bishop D. Factors modulating post-activation potentiation and its effect on performance of subsequent explosive activities. Sports Med. 2009;39(2):147-66. Eng J. Sample size estimation: how many individuals should be studied? Radiology. 2003 May;227(2):309-13.

13. Eng J. Sample size estimation: how many individuals should be studied? Radiology. 2003;227(2):309-13 14. Brown LE. Treinamento de força. Barueri, SP: Manole; 2008.

15. McArdle WD, Katch FI, Katch VL. Fisiologia do exercício: energia, nutrição e desempenho humano. 4a. ed. Rio de Janeiro: Guanabara-Koogan; 1998

16. Hermens HJ, Freriks B, Disselhorst-Klug C, Rau G. Development of recommendations for SEMG sensors and sensor placement procedures. J Electromyogr Kinesiol. 2000;10(5):361-74.

17. Winter DA. Biomechanics and motor control of human movement. New York: Wiley; 1990

18. Dowling JJ, Vamos L. Identification of kinetic and temporal factors related to vertical jump performance. J Appl Biomech. 1993:9:95-110.

19. Marchetti PH, Duarte M. Eletromiografia: uma breve revisão sobre os procedimentos de aquisição do sinal. Terapia Manual. 2011;9(44):548-53.

20. Rhea MR. Determining the magnitude of treatment effects in strength training research through the use of the effect size. J Strength Cond Res. 2004;18(4):918-20.

21. Marchetti PH, Matos VJP, Soares EG, Silva JJ, Serpa EP, Corrêa DA, et al. Can the technique of knee wrap placement affect the maximal isometric force during back squat exercise? Int J Sports Sci. 2015;5(1):16-8

22. Lowery RP, Duncan NM, Loenneke JP, Sikorski EM, Naimo MA, Brown LE, et al. The effects of potentiating stimuli intensity under varying rest periods on vertical jump performance and power. J Strength Cond Res. 2012;26(12):3320-5.

23. McCann MR, Flanagan SP. The effects of exercise selection and rest interval on postactivation potentiation of vertical jump performance. J Strength Cond Res. 2010;24(5):1285-91.

24. Crewther BT, Kilduff LP, Cook CJ, Middleton MK, Bunce PJ, Yang GZ. The acute potentiating effects of back squats on athlete performance. J Strength Cond Res. 2011;25(12):3319-25.

25. Seitz $L B$, de Villarreal ES, Haff GG. The temporal profile of postactivation potentiation is related to strength level. J Strength Cond Res. 2014;28(3):706-15. 